\title{
Uso de álcool, tabaco e drogas por jovens e adultos da cidade de Recife
}

\author{
Use of alcohol, tobacco and drugs among youngsters \\ and adults of the city of Recife
}

\author{
Nemésio Dario Almeida
}

Perito do Trânsito, Psicólogo Clínico e do Centro de Atenção Psicossocial (CAPS) Recife-PE, Mestre em Psicologia Social e da Personalidade pela Pontifícia Universidade Católica do Rio Grande do Sul (PUC-RS), Doutorando em Psicologia Cognitiva pela Universidade Federal de Pernambuco (UFPE), Recife, PE - Brasil, e-mail: nemesiodario@hotmail.com

\section{Resumo}

Este estudo teve por objetivo investigar o uso de álcool, tabaco e drogas por jovens e adultos do Centro de Atenção Psicossocial para Álcool e Drogas (CAPS-ad) da cidade de Recife. A amostra foi composta por 134 respondentes, de 12 a 65 anos. Para a coleta de dados, foram empregadas as informações colhidas no momento do processo de triagem e outras durante o desenvolvimento do tratamento. Os resultados apontam para um elevado consumo de álcool e tabaco, que também se destacam no consumo de outras substâncias. Quanto à história de consumo, amigos ou conhecidos são apontados na introdução ao uso, e também como companhia frequente para o consumo; como motivo de uso pela primeira vez destacam-se a curiosidade e a busca por diversão ou prazer.

Palavras-chave: Uso de drogas. Fatores de risco. Atenção psicossocial.

\begin{abstract}
This study aims to investigate the use of alcohol, tobacco and drugs among youngsters and adults of the Psychosocial Attention Center for Alcohol and Other Drugs (CAPS-ad), in the city of Recife. The sample was composed of 134 respondents from the age of 12 to 65 . The data used in this work were collected based on the information obtained with the selection process and during the development of the treatment. The results show a high consumption of alcohol and tobacco, which are also used when other substances are consumed. Regarding the history of the use, users
\end{abstract}


indicate friends or acquaintances as the ones responsible for starting them in the habit - they are also a frequent company for the practice. The main reasons for trying drugs for the first time are curiosity, fun or pleasure.

Keywords: Use of drugs. Risk factors. Psychosocial attention.

\section{Introduçáo}

O consumo de substâncias psicoativas (lícitas e ilícitas) constitui um grave problema de saúde pública, com complicações que podem atingir a vida pessoal, familiar, escolar, ocupacional e social do usuário (Almeida, 2004; Galduróz, Noto, Nappo \& Carlini, 2003; Nicastri \& Ramos, 2001; Tavares, Béria \& Lima, 2004). Em 1989, de 205.363 pessoas internadas por uso de drogas em hospitais psiquiátricos brasileiros, 95,6\% (196.324) foram devido ao álcool e 4,4\% (9.039) pelas demais drogas (Ministério da Saúde, 1991).

Pesquisas realizadas em vários países demonstram que a introdução ao consumo de substâncias psicoativas tem ocorrido cada vez mais cedo e que tal uso também ocorre de forma cada vez mais intensa. Em nosso país, certos estudos vêm corroborar tais descobertas - um exemplo é o que foi realizado pelo Centro Brasileiro de Informações sobre Drogas Psicoativas (CEBID, 2003) a respeito do uso indevido de drogas por estudantes dos antigos $1^{\circ}$ e $2^{\circ}$ graus, em dez capitais brasileiras (Galduróz, Noto \& Carlini, 1997).É durante a adolescência, com o desejo de serem livres da família, que as drogas costumam ser utilizadas por muitos jovens, muitos por curiosidade. Diversos estudos têm demonstrado que a grande maioria dos que virão a tornar-se dependentes usou drogas pela primeira vez entre os 14 e os 18 anos (Baus, Kupek \& Pires, 2002; KerrCorrêa, Andrade, Bassit \& Boccuto, 1999; Ribeiro, Pergher \& Torossian, 1998; Tavares, Béria \& Lima, 2001; Zaitter, 1994).

Para os teóricos da adolescência, essa é uma fase de rápido desenvolvimento e sucessão de eventos. Modificações importantes ocorrem no corpo, na mente, nos relacionamentos e na visão de mundo, em um breve espaço de tempo, oportunizando uma maior aproximação a fatores de risco, os quais podem favorecer, por exemplo, o envolvimento no uso de substâncias psicoativas. Para Kazdin (1993) os adolescentes não possuem ainda a habilidade para pressupor os perigos de um comportamento habitual, o que os torna mais expostos ao envolvimento em comportamentos de risco, como o uso e abuso de drogas, especialmente quando apresentam comportamento agressivo e impulsivo. Em muitos casos é possível localizar dificuldades emocionais, tais como relacionamento familiar abalado, conflitos internos, crise existencial e não aceitação de normas, entre as situações de risco no que diz respeito ao abuso dessas substâncias. A curiosidade quanto aos efeitos e sensações que as drogas propiciam e o exibicionismo, visto como autoafirmação de identidade, também são situações de perigo.

A família possui forte contribuição, tanto como amparo quanto como agente no uso de drogas pelos filhos. Segundo Chiapetti (2001) e Kazdin (1993), o arranjo e a dinâmica familiar em desarmonia aumentam a fragilidade do adolescente, tornando-o mais suscetível a condutas de risco tais como o uso de substâncias psicoativas, particularmente durante a pré-adolescência e adolescência.

Constata-se que o risco se eleva quando os pais são separados ou quando os jovens residem com outras pessoas. O desarranjo familiar e a ligação com colegas delinquentes, na adolescência, são intensos fatores para o surgimento do consumo de drogas. Além disso, adolescentes de pais consumidores ou dependentes de alguma droga são levados para o mesmo comportamento.

De outra forma, adolescentes cujos pais proporcionam normas bem definidas, e ao mesmo tempo incentivam seus filhos a exercerem sua liberdade de forma responsável, levando em conta o que a idade deles permite, de alguma maneira estão mais protegidos de comportamentos de risco, inclusive o uso de substâncias (Jessor, 1993). Famílias constituídas por ambos os pais tendem a ser mais protetoras (Dougherty, 1993; Jessor, 1993; Zaslow \& Takanishi, 1993). Um grande incremento familiar de proteção em relação ao consumo de substâncias psicoativas e outros comportamentos de risco é uma relação saudável entre os pais e os filhos, seja ela uma relação 
afetuosa, o tempo passado com os filhos, a firmeza de medidas disciplinares assumidas ou a abstenção no uso de drogas (Baus, Kupek \& Pires, 2002; Brook et al., 1998).

A comunidade também imprime proteção e riscos ao adolescente. No campo comunitário, a escola é o ambiente de grande influência. Adolescentes que se sentem parte integrante da comunidade escolar, e que contam com apoio positivo dos pais com relação ao seu desempenho na escola, estão mais imunes a comportamentos de risco (Jessor, 1993). Um outro fator comunitário de ação de amparo e agente de riscos é o grupo de amigos. De acordo com Zaitter (1994), a turma de amigos é um conjunto de indivíduos com os mesmos interesses e anseios. O autor destaca ainda que a presença dos pais na amizade dos filhos e o entendimento da natureza das aproximações feitas por eles (ao invés de simplesmente coibi-las ou entendê-las como uma fase) são fatores de amparo. Da mesma forma, estudos têm demonstrado que o uso tem início pelo fato de o adolescente ter colegas que consomem drogas, produzindo uma pressão do grupo na direção do consumo (Pechansky, Szobot \& Scivoletto, 2004).

Não devemos esquecer que a mídia é um dos espaços de grande persuasão comunitária sobre o comportamento das pessoas. O uso de drogas, principalmente de álcool e cigarros, encontra-se presente, e é continuamente estimulado, em anúncios comerciais, filmes, letras de música e outros meios de comunicação de massa. A apresentação dessas substâncias associadas a fatores desejáveis como prazer, beleza, sucesso financeiro e sexual, poder e outros, de forma explícita ou implícita, configura-se como um importante elemento de risco para o seu consumo (Jessor, 1993; Zaslow \& Takanishi, 1993).

Dessa forma, a mídia, ao mesmo tempo em que nos informa, muitas vezes ultrapassa a sua responsabilidade social, tornando-se fonte de muitos equívocos e desinformações, graças ao seu comprometimento com o mercado de anunciantes. Assim, MacRae e Simões (2000) chamam a atenção para o papel exercido pela imprensa a partir de meados dos anos 1950, enfatizando o lugar de "desordeiro" e de "desvio de caráter" atribuído às pessoas que usavam maconha. Nesse particular, o papel exercido pela mídia foi mais intenso do que as revelações das pesquisas científicas da época. Isso foi em grande parte responsável pela maneira como as novas gerações foram instruídas sobre esse assunto. Outro aspecto foi a introdução de motivos infantis, tais como lebre, tartaruga e siris, nas propagandas de bebidas alcoólicas, num claro apelo dirigido ao público jovem.

Quando nos referimos ao uso de substâncias no Brasil e em outros países, diversos trabalhos têm demonstrado que o álcool é a substância mais usada pelos jovens, seguida pelo tabaco, maconha e estimulantes. Os solventes são populares principalmente entre os adolescentes mais novos (Almeida, 2004).

Um desses trabalhos, conduzido por Baus, Kupek e Pires (2002) entre alunos de $1^{\circ}$ e $2^{\circ}$ graus de uma escola de Florianópolis, em 2002, demonstrou um aumento do risco em alunos com idade superior a 15 anos, sendo de três vezes para o uso de cocaína, mais de cinco para alucinógenos, mais de sete para a maconha e mais que o dobro para tranquilizantes. No que se refere ao gênero, as mulheres demonstraram possuir o dobro de chance de fazerem uso de medicamentos para emagrecer ou ficar acordadas, e um pouco mais que o dobro de chance de usar tranquilizantes. Todavia, manifestaram risco duas vezes menor de uso de solventes. $\mathrm{O}$ índice de uso de álcool foi 10\% menor em alunos de classe C e D do que em alunos de classe A e B, e o consumo de cigarro e maconha foi maior entre alunos que residiam com outras pessoas sem ser os pais. Também ficou demonstrado um elevado consumo de álcool e tabaco, sendo a utilização da primeira substância maior nas classes socioeconômicas mais altas. Quanto às drogas ilícitas, verificou-se maior consumo na classe média. Percebeu-se também uma associação entre o uso de cocaína e crack, e entre alucinógenos e solventes, sendo o risco quase quatro vezes maior nas classes socioeconômicas mais baixas (Baus, Kupek \& Pires, 2002). Fortalecendo esses resultados, em um outro estudo realizado na cidade de Cuiabá entre estudantes do ensino fundamental e médio da rede pública, Souza e Martins (1998) comprovaram que a primeira substância de consumo entre as drogas arroladas em seu estudo foi o álcool, e a segunda, o tabaco.

Segundo Bortoletto (1990), o alcoolismo é um problema social - ele ressalta que sua gravidade pode ser verificada por meio das estatísticas de agressões, violência no trânsito, absenteísmo e acidentes de trabalho, eventos sempre relacionados ao consumo de álcool. Esse ponto de vista é corroborado por Souza e Martins (1998), que lembram que o alcoolismo é a mais frequente das toxicomanias em todo o mundo.

Deve-se ressaltar que a implantação de programas de prevenção ao uso de álcool, tabaco 
Almeida, N. D.

e drogas demanda o conhecimento da realidade do consumo, incluindo aspectos relacionados ao consumo geral e em populações específicas, bem como no que diz respeito aos tipos de drogas consumidos, e ainda indicadores estatísticos sobre as suas consequências (hospital, mortes, apreensões, entre outras). A pesquisa da qual se deriva esse relato teve por objetivo investigar o consumo de álcool, cigarros e drogas por jovens e adultos da cidade de Recife, bem como tipos de substâncias consumidas e fatores relacionados à história de consumo.

\section{Procedimento metodológico}

\section{Participantes}

As informações obtidas referentes aos dados sociodemográficos dos usuários, com a frequência e percentual de ocorrência respectivos, estão ilustradas na Tabela 1. A amostra é composta na sua maioria pelos usuários do sexo masculino, que compõem $76,1 \%$ dos respondentes, contra $23,8 \%$ do sexo feminino (ou seja, 102 homens e 32 mulheres). Isso vem corroborar os estudos feitos anteriormente, que constatam também o uso predomintante de drogas por homens. Constata-se que a principal faixa etária está entre 12 a 17 anos de idade (34,2\%), seguida de 18 a 28 anos (20,9\%), caracterizando uma amostra pré-adolescente e adolescente para o uso de drogas, indo ao encontro de estudos que vêm confirmando a tendência precoce no uso de substâncias psicoativas. Quanto ao nível socioeconômico, a maioria dos usuários $(72,3 \%)$ estão em nível baixo e, em relação à escolaridade, grande parte dos usuários $(82,8 \%)$ possui o ensino fundamental incompleto.

\section{Procedimentos}

Para a coleta de dados, foram empregadas as informações colhidas no momento do processo de triagem e outras obtidas durante o desenvolvimento do tratamento dos dependentes químicos que estavam em atendimento durante o ano de 2005, em um Centro de Atenção Psicossocial para Álcool e Drogas (CAPS-ad) na cidade do Recife. Após o levantamento de todos os prontuários, houve a análise dos dados obtidos quanto à definição sociodemográfica da amostra e ao percentual de ocorrência relativo

Tabela 1 - Uso de álcool, tabaco e drogas por jovens e adultos da cidade de Recife - Frequência e percentual de ocorrência relativos aos dados sócio-demográficos da amostra $(n=134)-2005$

(Continua)

\begin{tabular}{lcc}
\hline \multicolumn{1}{c}{ Sexo } & $f$ & $\%$ \\
\hline Feminino & 32 & 23,8 \\
Masculino & 102 & 76,1 \\
Total & 134 & $\mathbf{1 0 0 , 0}$ \\
\hline \multicolumn{1}{c}{ Idade } & \multicolumn{2}{c}{$\%$} \\
\hline Entre 12 e 17 anos & 46 & 34,2 \\
Entre 18 e 28 anos & 28 & 20,9 \\
Entre 29 e 40 anos & 24 & 17,9 \\
Entre 41 e 50 anos & 20 & 14,9 \\
Entre 51 e 60 anos & 13 & 9,7 \\
Mais de 61 anos & 3 & 2,2 \\
Total & 134 & $\mathbf{1 0 0 , 0}$ \\
\hline
\end{tabular}


Tabela 1 - Uso de álcool, tabaco e drogas por jovens e adultos da cidade de Recife - Frequência e percentual de ocorrência relativos aos dados sócio-demográficos da amostra $(n=134)-2005$

(Conclusão)

\begin{tabular}{lcc}
\hline \multicolumn{1}{l}{ Nível socioeconômico } & $\boldsymbol{f}$ & $\%$ \\
\hline Baixo & 97 & 72,3 \\
Médio & 37 & 27,6 \\
Total & $\mathbf{1 3 4}$ & $\mathbf{1 0 0 , 0}$ \\
\hline \multicolumn{1}{c}{ Escolaridade } & \multicolumn{2}{c}{$\%$} \\
\hline Analfabeto & \multicolumn{2}{c}{$\%$} \\
Ensino fundamental & \multicolumn{2}{c}{2,9} \\
Ensino médio & 111 & 82,8 \\
Ensino superior & 18 & 13,4 \\
Total & 1 & 0,7 \\
\hline
\end{tabular}

aos dados por uso isolado e por consumo múltiplo de drogas da amostra, os quais estão ilustrados nas Tabelas 2 e 3.

Em relação aos procedimentos éticos, os participantes receberam um formulário contendo os objetivos da pesquisa, bem como o consentimento informado para sua participação. Não ocorreram recusas na participação. Além disso, todos os participantes assinaram o termo de consentimento livre e esclarecido, assegurando o anonimato e os preceitos em pesquisa.

\section{Resultado e discussáo}

Quando falamos em consumo de drogas, sabemos que ele acompanha a história da humanidade, estando presente durante todo o processo civilizatório. As circunstâncias desse consumo, no entanto, variaram bastante ao longo dos tempos, e ainda hoje são diferentes dependendo do contexto em que tal uso ocorre.

Seguindo a trajetória histórica dos povos, notamos que o consumo de drogas foi adquirindo características próprias, ora privilegiando o uso ritualístico e religioso, ora o uso medicinal, ora o uso agregador e, mais recentemente, a contestação. Para Escohotado (1996, citado por Melcop, 2004), a história das drogas deu luz própria à história da humanidade, tendo influenciado não só a evolução da medicina, mas também a evolução da moral, da religião, da economia e da política.

A utilização de substâncias psicoativas nas sociedades modernas reflete as importantes transformações sociais e econômicas dos últimos séculos, que ocasionaram mudança em muitos costumes e deslocaram os mecanismos de controle comunitário para as grandes corporações e instituições anônimas. No polo oposto ao do vegetal transformado artesanalmente em substância psicoativa, temos hoje a droga em série, produto de transformação laboratorial e produzida, lícita ou clandestinamente, em escala repetitiva como qualquer outro bem de consumo generalizado.

Assim, os tempos modernos fizeram surgir novas motivações e novas formas de obtenção de substâncias psicoativas, tanto por jovens como por adultos de todas as classes sociais. O álcool talvez seja uma das substâncias psicoativas mais utilizadas em todo o mundo - dependendo da dose, frequência e circunstâncias, ele pode ser consumido sem problemas. No entanto, levantamentos recentes indicam que uma fração importante da população substitui esse use recreativo por outros, chamados de risco ou prejudicial, que podem acarretar graves consequências físicas, psicológicas e sociais.

No que diz respeito ao presente artigo, e em se tratando de aspectos relacionados à história 
do uso de drogas ou álcool pelos usuários, constata-se que a introdução ao uso se deu por influência de "colegas, amigos ou conhecidos", sendo estes também indicados como companhia frequente para o uso de drogas. O motivo do uso pela primeira vez foi fundamentalmente a busca por "diversão ou prazer" ou, ainda, "curiosidade". Esses resultados são muito semelhantes àqueles apontados por Kerr-Corrêa, Andrade, Bassit e Boccuto (1999), que realizaram uma pesquisa dessa natureza com estudantes do curso de Medicina da Unesp.

Os motivos mencionados para o uso frequente de drogas ou álcool são principalmente a "quebra de rotina" e "para curtir efeitos destas substâncias", seguidos pela "diminuição da ansiedade ou estresse". Esses motivos foram igualmente predominantes em pesquisa similar realizada por KerrCorrêa et al. (2001), também com alunos da Unesp.

A Tabela 2 mostra o resultado em que o álcool é a droga mais usada pelos dependentes de substâncias psicoativas, totalizando $54 \%$ desses usuários. O tabaco aparece em segundo lugar em termos de consumo, com 33\% dos usuários.

$\mathrm{Na}$ Tabela 3, referimo-nos ao comportamento dos dependentes que usam múltiplas drogas, ou seja, a associação de uso de duas ou mais drogas diferentes pelo usuário: o tabaco vem em primeiro lugar, com $35 \%$ dos usuários, seguido pelo consumo de álcool com $23 \%$ e a maconha com $21 \%$, em terceiro lugar.

Tabela 2 - Uso de álcool, tabaco e drogas por jovens e adultos da cidade de Recife - Percentual de ocorrência referente aos dados por uso isolado de drogas da amostra $(n=134)-2005$

\begin{tabular}{|c|c|}
\hline Tipos de drogas & $\%$ \\
\hline Álcool & 54,0 \\
\hline Tabaco & 33,0 \\
\hline Maconha & 8,0 \\
\hline Inalantes & 5,0 \\
\hline Total & 100,0 \\
\hline
\end{tabular}

Tabela 3 - Uso de álcool, tabaco e drogas por jovens e adultos da cidade de Recife - Percentual de ocorrência referente aos dados por consumo múltiplo de drogas da amostra $(\mathrm{n}=134)-2005$

\begin{tabular}{|c|c|}
\hline Tipos de drogas & $\%$ \\
\hline Tabaco & 35,0 \\
\hline Álcool & 23,0 \\
\hline Maconha & 21,0 \\
\hline Cocaína & 10,0 \\
\hline Crack & 7,0 \\
\hline Inalantes & 3,0 \\
\hline Ansiolíticos & 1,0 \\
\hline Total & 100,0 \\
\hline
\end{tabular}


O consumo observado, tanto no que diz respeito a álcool e tabaco quanto a drogas ilícitas, apresenta-se em níveis próximos àqueles descritos em estudos realizados com jovens de outras cidades do Brasil. Dessa forma, diante dos resultados obtidos nesta pesquisa, e sendo o consumo de substâncias psicoativas (lícitas e ilícitas) um problema social e de saúde pública, percebe-se a importância da realização de programas de prevenção e intervenção nessa área.

Uma parcela da população especialmente suscetível a esse problema, como já foi mencionado, são os jovens. Poder-se-ia supor, de modo genérico, que há uma homogeneidade nesse grupo quanto à realidade de consumo, o que apontaria para estratégias de prevenção e intervenção também homogêneas. Entretanto, como mostram os resultados desta e de outras pesquisas da mesma natureza realizadas com a população jovem, existem especificidades no consumo, que se referem à quantidade e tipo de droga consumida, entre outros fatores, o que aponta para a complexidade da relação entre o consumo dessas substâncias e o meio onde o usuário se insere. Tal complexidade aponta para a necessidade de se considerar esses fatores em programas de intervenção e prevenção ao uso e abuso de substâncias.

É importante também que resgatemos a função social da mídia. Embora tenha se transformado em um negócio que envolve milhões de dólares, a mídia, assim como qualquer outro negócio, deve assumir sua responsabilidade social - como fez, por exemplo, na prevenção da infecção pelo HIV e na redução do preconceito em relação à aids. Tem sido muito pouco considerada a colaboração que a mídia poderia dar no encaminhamento das questões associadas ao uso de drogas - legais ou ilegais.

A mídia já reconheceu a responsabilidade social de outros tipos de negócios, mas se recusa a aceitar a sua própria responsabilidade quando se trata de ajudar a sociedade no que se refere às questões como os danos relativos ao consumo de álcool. Ao informar, sugerir soluções e estratégias, e provocar discussões, a mídia estaria mostrando que está ciente de suas responsabilidades para com a sociedade que nós mesmos criamos. Por fim, hoje sabemos que a qualidade de vida e, consequentemente, a saúde são influenciadas muito mais pela política e pela distribuição de terra e riquezas do que pelo tratamento e prevenção de doenças, que também não deixam de ser importantes.

\section{Consideraçóes finais}

Este estudo foi realizado com o objetivo de detectar "o uso de álcool, tabaco e drogas por jovens e adultos da cidade de Recife", motivado por uma demanda espontânea. O resultado encontrado apontou para uma prevalência de 54\% de ocorrência no que refere aos dados por uso isolado de drogas e de $35 \%$ de ocorrência referente aos dados por consumo múltiplo de drogas.

Esses resultados demonstram a necessidade urgente de programas específicos para detecção e tratamento precoces para o consumo de drogas. Faz-se necessário treinar recursos humanos envolvidos direta ou indiretamente com a assistência, o que poderá possibilitar o diagnótico precoce e, consequentemente, uma intervenção global junto a esses indivíduos. Acreditamos que, se detectado precocemente, o profissional terá maior chance de intervir, mesmo sem ter havido ainda um comprometimento a nível social e biológico para o depentente.

Por outro lado, recomenda-se que sejam desenvolvidos programas de orientação e esclarecimento junto a vários segmentos sociais (escolas, universidades, comunidades, etc.) no sentido de prevenir a dependência, bem como detectar e encaminhar para tratamento possíveis indivíduos com problemas relacionados ao uso e abuso de drogas.

\section{Referências}

Almeida, N. D. V., \& Dias, M. R. (2004). O consumo de bebidas alcoólicas na condução de veículos: Um estudo de crenças. Revista Symposium, 8(1), 24-37.

Baus, J., Kupek, E., \& Pires, M. (2002). Prevalência e fatores de risco relacionados ao uso de drogas entre escolares. Revista de Saúde Pública, 36(1), 40-46.

Bortoletto, M. E. (1990). Tóxicos, civilização e saúde: Contribuição à análise dos sistemas de informações tóxico-farmacológicas no Brasil. Rio de Janeiro: Fundação Oswaldo Cruz.

Brook, J. S., Brook, D. W., De La Rosa, M., Duque, L. F., Rodríguez, E., Montoya, I. D. et al. (1998). Pathways to marijuana use among adolescents: Cultural/ ecological, family, peer, and personality influences. Journal of American Academy of Child \& Adolescent Psychiatry, 37, 759-766. 
Centro de Estudos em Biodireito - CEBID. (2003). Livreto informativo sobre drogas psicotrópicas. São Paulo: Cromosete.

Chiapetti, N. (2001). Comportamento de risco em pré-adolescentes institucionalizados. Tese de Doutorado, Curso de Pós-Graduação em Psicologia Clínica, Instituto de Psicologia, Universidade de São Paulo, SP.

Dougherty,D. M. (1993). Adolescent health: Reflections on a repport to U.S. Congress. American Psychologist, 48(2), 193-201.

Galduróz, J. C. F., Noto, A. R., \& Carlini, E. A. (1997). Quarto levantamento sobre o uso de drogas entre estudantes de $1^{\circ}$ e $2^{\circ}$ graus em 10 capitais brasileiras - 1997. São Paulo: CEBRID.

Galduróz, J. C. F., Noto, A. R., Nappo, S. A., \& Carlini, E. A. (2003). Comparações dos resultados de dois levantamentos domiciliares sobre o uso de drogas psicotrópicas no estado de São Paulo nos anos de 1999 e 2001. Jornal Brasileiro de Psiquiatria, 52(1), 43-51.

Jessor, R. (1993). Successful adolescent development among youth in high-risk settings. American Psychologist, 48(2), 117-126.

Kazdin, A. E. (1993). Adolescent mental health: Prevention and treatment programs. American Psychologist, 48(2), 127-141.

Kerr-Corrêa, F., Andrade, A. G., Bassit, A. Z., \& Boccuto, N. M. V. F. (1999). Uso de álcool e drogas por estudantes de medicina da UNESP. Revista Brasileira de Psiquiatria, 21(2), 95-100.

Kerr-Corrêa, F., Dalben, I., Trinca, L. A., Simão, M. O., Mattos, P. F., Ramos-Cerqueira, A. T. A. et al. (2001). Primeiro levantamento do uso de álcool e de drogas e das condições gerais de vida dos estudantes da UNESP - 1998. (Série Pesquisa Vunesp, 14). São Paulo: Fundação para o Vestibular da Universidade Estadual Paulista.

MacRae, E., \& Simões, J. A. (2000). Rodas de fumo: Uso de maconha entre as camadas médias urbanas. Salvador: Ed. da UFBA.
Melcop, A. G. (2004). Vamos parar por aqui? Os desafios da abordagem de redução de danos nas violências no trânsito. In E. Buning, M. Gorgulho, A. G. Melcop \& P. O'Hare (Org.). Álcool e redução de danos: Uma abordagem inovadora para países em transição. Brasília: Ministério da Saúde.

Ministério da Saúde. (1991). Normas e procedimentos na abordagem do abuso de drogas. Brasília: SNAS, DPS, CORSAM.

Nicastri, S., \& Ramos, S. D. P. (2001). Prevenção do uso de drogas. Revista ABEAD-JBDQ, 2(1), 25-29.

Pechansky, F., Szobot, C. M., \& Scivoletto, S. (2004). Uso deálcool entre adolescentes: Conceitos, características epidemiológicas e fatores etiopatogênicos. Revista Brasileira de Psiquiatria, 26(1), 14-17.

Ribeiro, T. W., Pergher, N. K., \& Torossian, S. D. (1998). Drogas e adolescência: Uma análise da ideologia presente na mídia escrita destinada ao grande público. Psicologia: Reflexão e Crítica, 11(3), 421-430.

Souza, D. P. O., \& Martins, D. T. O. (1998). O perfil epidemiológico do uso de drogas entre estudantes de $1^{\circ}$ e $2^{\circ}$ graus da rede estadual de ensino de Cuiabá. Cadernos de Saúde Pública, 14(2), 391-400.

Tavares, B. F., Béria,J. U., \& Lima, M. S. (2001). Prevalência do uso de drogas e desempenho escolar entre adolescentes. Revista de Saúde Pública, 35(2), 150-158.

Tavares, B. F., Béria, J. U., \& Lima, M. S. (2004). Fatores associados ao uso de drogas entre adolescentes escolares. Revista de Saúde Pública, 38(6), 787-796.

Zaitter, M. A. B. (1994). Drogadição: Drogas e conseqüências. São Paulo: Lovise.

Zaslow, M. J., \& Takanishi, R. (1993). Priorities for research on adolescent development. American Psychologist, 48(2), 185-192.

Recebido: 18/05/2009 Received: 05/18/2009

Aprovado: 15/09/2009 Approved: 09/15/2009 